\title{
Effects of Osteotomy Angle on Tibial Angulation and Torsion During CORA-Based Leveling Osteotomy in Toy Breed Dogs: A Computer Modeling-Based Study
}

\author{
Youngeun Jeong ${ }^{\dagger}$, Jaemin Jeong ${ }^{\dagger}$, Cheongwoon Cho, SeongMok Jeong and Hae Beom Lee ${ }^{1}$ \\ College of Veterinary Medicine, Chungnam National University, Daejeon 34134, Korea
}

(Received: March 31, 2020 / Revised: July 01, 2020 / Accepted: August 03, 2020)

\begin{abstract}
The objective of this study was to determine the effect of osteotomy angle and tibial proximal segment rotation angle on angular and torsional tibial deformities and to assess the trends of these deformities during the rotation of the tibial proximal segment in a center of rotation of angulation (CORA)-based leveling osteotomy (CBLO) by performing computer modeling of the tibia. Four tibias of toy breed dogs with no history of lameness were used in this study. Osteotomies were performed in the proximal tibias at angles of $0^{\circ}, 10^{\circ}, 20^{\circ},-10^{\circ}$, and $-20^{\circ}$, perpendicular to either the proximodistal or craniocaudal tibial axes. The mechanical medial proximal tibial angle (mMPTA) and transcondylar (TC) and distal cranial tibial ( $\mathrm{CnT}$ ) axes were used to measure angular and torsional deformities, respectively. All tibias showed an increase in angular and rotational deformities with an increase in the tibial plateau rotation angle. The tibia with osteotomies performed in the proximodistal and craniocaudal directions showed the highest magnitude of torsional and angular deformities, respectively. The results of this study revealed a tendency of occurrence of angular and torsional deformities with osteotomy performed along the proximodistal and craniocaudal directions in the CBLO.
\end{abstract}

Key words : center of rotation of angular (CORA)-based leveling osteotomy, bone deformity, computer modeling, cranial cruciate ligament, dog.

\section{Introduction}

Cranial cruciate ligament (CCL) injury is one of the most common orthopedic injuries of hindlimbs in dogs $(3,4,8$, $9,19)$. Injury of the CCL allows cranial translation of the tibia, resulting in stifle instability and osteoarthritis $(18,19)$. Dynamic stabilization of the CCL-deficient joint that neutralizes cranial tibial thrust is recommended by most veterinary surgeons because of the predisposition of this joint to degenerative changes $(6,7,11,12,14-16)$. Among these surgeries, proximal tibial leveling osteotomy based on the center of rotation of angulation (CORA), which alters mechanics and reduces cranial tibial thrust associated with the CCL-deficient stifle, has been implemented recently $(10,13)$. The CORA-based leveling osteotomy (CBLO) is a procedure in which the osteotomy is centered at the CORA, and correction of the CORA magnitude results in the desired tibial plateau angle (TPA) through alignment of the proximal and distal anatomic longitudinal axis.

CBLO has unique advantages relative to other osteotomy techniques, such as tibial plateau leveling osteotomy (TPLO), tibial tuberosity advancement (TTA), and cranial wedge ostectomy (CWO). This technique preserves the proximal tibial epiphysis, which allows the application of ancillary stabilization and intra- or extra-articular procedures $(10,13,17)$.

\footnotetext{
These authors contributed equally to this work.

Corresponding author.

E-mail : seatiger76@cnu.ac.kr
}

Because the axis of correction is centered on the CORA, this procedure allows the positioning of the center of the proximal tibial epiphysis on the tibial shaft. Therefore, there is no secondary translation and 'balcony' effect as seen with TPLO (10).

However, CBLO may lead to the development of angular or torsional deformity after reduction during surgery. Previous studies have reported similar complications with TPLO (20). Tibial osteotomy has been recommended to be performed parallel to the tibial plateau (16). Because of the anatomical differences among dogs, the tibial long axis and the tibial plateau may not be parallel. Therefore, the angle that is made at the osteotomy is not truly parallel to the joint surface or perpendicular to the sagittal plane of the tibia (21).

One study described the effect of the osteotomy angle on the alteration of the tibial plateau rotation angle for TPLO (21). In tibial osteotomies performed at $20^{\circ}$ angle along the craniocaudal and proximodistal directions, maximum deformities of about $15^{\circ}$ in the valgus and $10^{\circ}$ in the external rotation, respectively, were noted. The osteotomy angles that showed the least variation from $0^{\circ}$ for rotational and angular deformity should be considered optimal for use in a clinical setting, and several osteotomy angles should be eliminated.

The objective of this study was to describe the effect of the osteotomy angle and tibial proximal segment rotation angle on angular and torsional tibial deformities and to assess the trends of these deformities during rotation of the tibial proximal segment in the CBLO by using computer modeling. We hypothesized that angular and torsional limb deformities after 
the CBLO procedure may result from an osteotomy that is not truly perpendicular to the craniocaudal axis or not parallel to the tibial plateau.

\section{Materials and Methods}

\section{D models}

Three-dimensional (3D) computer models of each unilateral tibia were based on computed tomographic (CT) data of toy breed dogs with no history of pelvic limb lameness that presented to the veterinary medical teaching hospital of Chungnam National University between January 2016 and August 2018. The side of the pelvic limbs was randomly assigned to be enrolled using a coin flip. The inclusion criteria were having a $\mathrm{CT}$ scan of appropriate quality to allow reconstruction into the $3 \mathrm{D}$ model and with a complete tibia, being skeletally mature, weighing $<5 \mathrm{~kg}$ and not having orthopedic diseases including fractures, osteoarthritis or neoplasia. The scan data were considered unacceptable when the thickness or orientation of the scan cut off or flattened the tibial condyle to an extent where accurate measurements could not be made. The breeds, sexes, body weights and ages of the patients were recorded.

Images were acquired using a CT scanner (Alexion $^{\mathrm{TM}}$, Tosiba, Japan) with a slice thickness of $1 \mathrm{~mm}$ and an overlapping increment of $0.8 \mathrm{~mm}$. In the resulting $\mathrm{CT}$ data set, the femur and tarsus were manually excluded to isolate the tibia, and $3 \mathrm{D}$ reconstruction of the tibia was performed using a 3D slicer (Slicer 4.10.2; open source software, www.slicer.org).

\section{Surgical planning}

Planning and surgical techniques were executed using a previously described method (13). The proximal tibial anatomic axis and distal tibial anatomic axis were determined
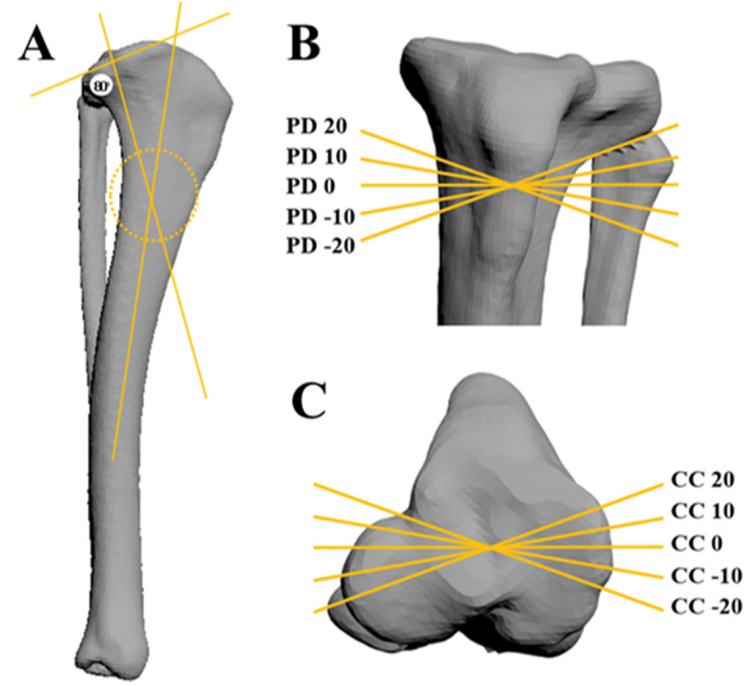

Fig 1. (A) CORA-based leveling osteotomy Preoperative planning: The desired postTPA of 10 is identified, and the CORA is defined by the intersection between the proximal and distal anatomic axes. The saw blade was positioned perpendicular to the tibial anatomic axis and parallel to the tibial plateau $(\mathrm{PD}=0$, $\mathrm{CC}=0$ ). Osteotomy angles made along the proximodistal axis (B) and craniocaudal axis (C). using 3ds MAX (3ds MAX 2020, Autodesk, Inc.; USA, www.autodesk.co.kr; Fig 1A). The intersection of these two axes constitutes the anatomic CORA. Radial osteotomy was centered over this point. The correction of the CORA magnitude resulted in a desired postoperative TPA (postTPA) of $10^{\circ}$. The diameter of the saw used in all tibia models was $8 \mathrm{~mm}$. The tibial proximal segment was rotated, such that the proximal and distal osteotomy surfaces were reduced without regard for alignment of the medial tibial cortices.

This tibia was assigned the notation of PD (proximodistal) $=$ 0 and $\mathrm{CC}$ (craniocaudal) $=0$, which indicates that the saw was at an angle of $0^{\circ}$, perpendicular to the proximal axis, and $0^{\circ}$, perpendicular to the craniocaudal axis. For the purpose of this study, the proximal and craniocaudal axes of the tibia were defined as being perpendicular to the tibial plateau and tibial mechanical axis, respectively. For all tibias, osteotomies with variations along the craniocaudal axis were perpendicular to the craniocaudal axis, $\mathrm{CC}=0$, and osteotomies with variation along the proximodistal axis were perpendicular to the proximodistal axis, $P D=0$. Negative-numbered angles represented that the saws were driven in a distal-toproximal or caudal-to-cranial direction at the medial aspect. Each tibia was simulated as follows: $\mathrm{PD}=0 / \mathrm{CC}=0, \mathrm{PD}=$ $10, \mathrm{PD}=20, \mathrm{PD}=-10, \mathrm{PD}=-20, \mathrm{CC}=10, \mathrm{CC}=20, \mathrm{CC}=$ -10 , and $\mathrm{CC}=-20$ (Fig 1B).

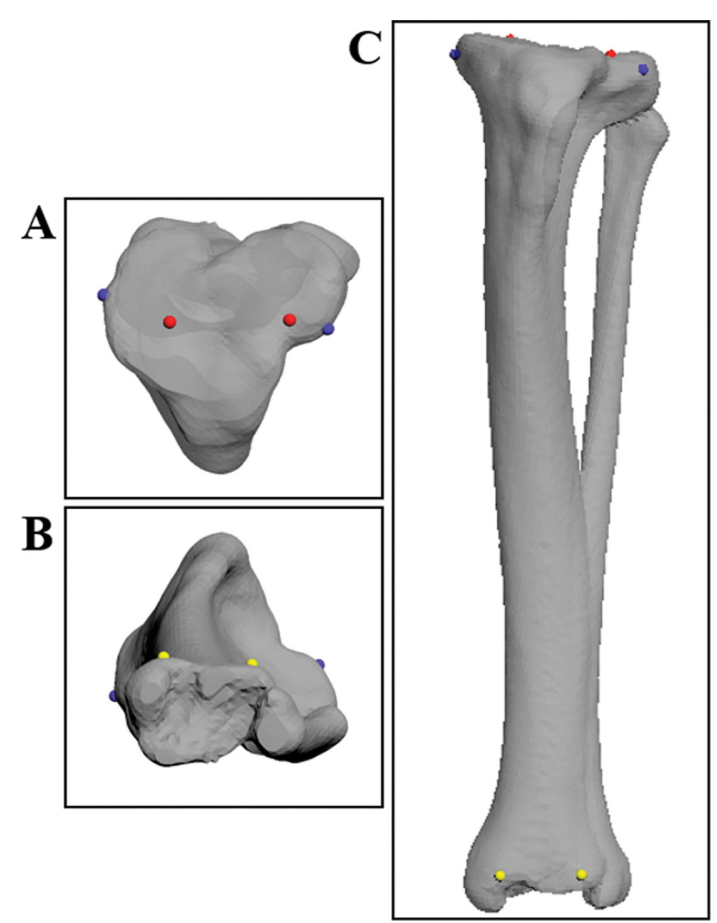

Fig 2. The 3D tibia model of a small dog. (A) Proximal articular surface of the tibia. Landmarks for the transcondylar (TC) axis represented by blue spheres marked on the caudo-lateral extent of the extensor sulcus and the prominence at the medial collateral ligament insertion (2). (B) Distal articular surface of the tibia. Landmarks for the distal cranial tibial $(\mathrm{CnT})$ axis represented by yellow spheres marked on the most cranial cortices of the distal tibia (2). (C) Frontal plane of the tibia. Landmarks for the proximal joint orientation line represented by red spheres marked on the most distal points of the medial and lateral tibial condyles (5). 


\section{Data collection}

The mechanical medial proximal tibial angle (mMPTA) and transcondylar (TC) and distal cranial tibial (CnT) axes were used to measure angular and torsional deformities, respectively. The angle between the mechanical axis and the joint orientation lines were measured on the proximomedial aspects to determine the MMPTA (5). The torsion angle, expressed in degrees, was calculated by determining the difference between the TC and $\mathrm{CnT}$ axes $(1,2)$. The X coordinate system was established for the tibial mechanical axis. The landmarks established for the mMPTA, TC, and CnT were attached $3 \mathrm{~d}$ tibia model before rotation of the tibial proximal segment after osteotomy (Fig 2).

Coordinate changes between landmarks for mMPTA, TC, and $\mathrm{CnT}$ on the tibia were used to calculate the magnitude of tibial angular and torsional deformity induced as the tibial proximal segment was rotated using $3 \mathrm{~d}$ MAX. For each of the nine tibias, five data collections were performed. Each test consisted of angular and torsional values recorded at $0^{\circ}$, $5^{\circ}, 10^{\circ}, 15^{\circ}, 20^{\circ}, 25^{\circ}$, and $30^{\circ}$ of tibial proximal segment rotation. Tibial proximal segment rotation was achieved automatically during data collection. Mean angular and torsional tibial deformity values for each tibial proximal segment rotation point were determined for each tibia to evaluate trends in the induced limb deformity with varying tibial proximal segment rotation and tibial osteotomy angles.

\section{Statistical analysis}

All the statistical analyses were performed using SPSS software version 24.0 (IBM SPSS Statistics 24.0, IBM Corp., Chicago, US). Descriptive data (e.g., means and standard deviation [SD]) were recorded for each outcome measure.

\section{Results}

Four dogs were enrolled during the study period. Breeds consisted of one German spitz, one Toy Poodle, one Maltese, and one Chihuahua. One dog was female, one dog was spayed female and two dogs were castrated males. Mean \pm SD age was $4.8 \pm 3.9$ years and body weight was $3.2 \pm 0.9$ $\mathrm{kg}$. Median (range) body condition score was 5 of 9 (4-7). Both two right and left tibia were used. Pre-experimental TPA was $27.4 \pm 2.2^{\circ}$.

Angular and torsional deformity varied depending on the degree of tibial proximal segment rotation and the osteotomy angle. A consistent trend existed for the tibial plateau rotation angle. The magnitude of angular and rotational deformity increased with an increase in the degree of tibial proximal segment rotation (Fig 3 and 4).

\section{Angular deformity}

The valgus worsened as the tibial proximal segment was rotated in the tibias with osteotomies performed in the caudal-to-cranial direction (CC, negative numbers; Fig 3). Tibias with osteotomies performed in the cranial-to-caudal direction (CC, positive numbers) showed varus deformity. Tibias with osteotomies performed in the proximal-to-distal (PD, positive number) and distal-to-proximal (PD, negative number) directions showed valgus and varus tendencies, respectively, but they were within the normal ranges (5). The tibias with osteotomies performed in the cranial-to-caudal and caudal-to-cranial directions showed the highest varus $\left(-9.75^{\circ}\right)$ and valgus $\left(9.95^{\circ}\right)$, respectively. A difference in the mMPTA of the tibia at $\mathrm{PD}=0 / \mathrm{CC}=0$ led to a minor change in the angle, which was in the range of $0.02^{\circ}$ to $0.10^{\circ}$.
A

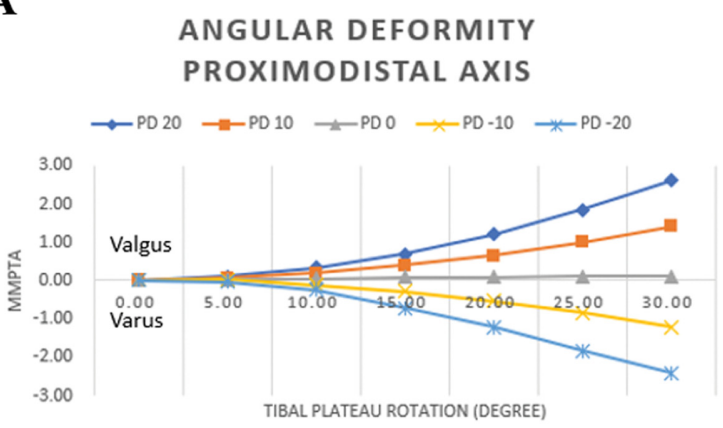

B

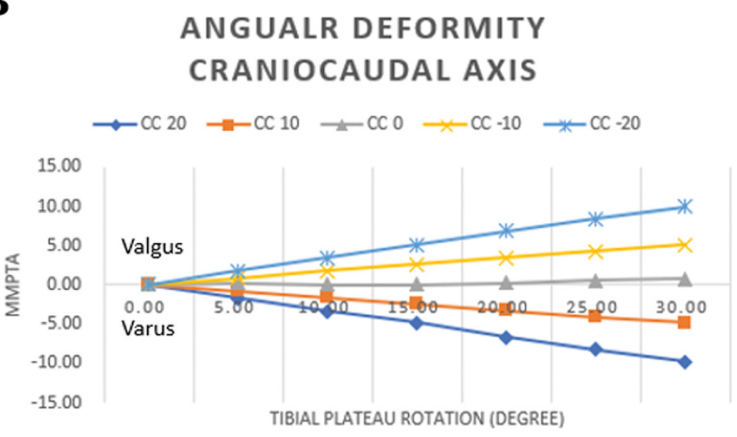

Fig 3. Angular deformities in tibias that underwent osteotomies in the proximodistal (A) and craniocaudal directions (B).

A
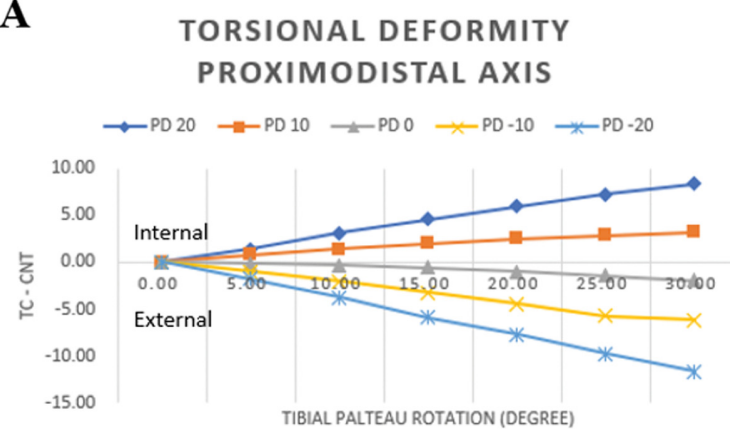

B

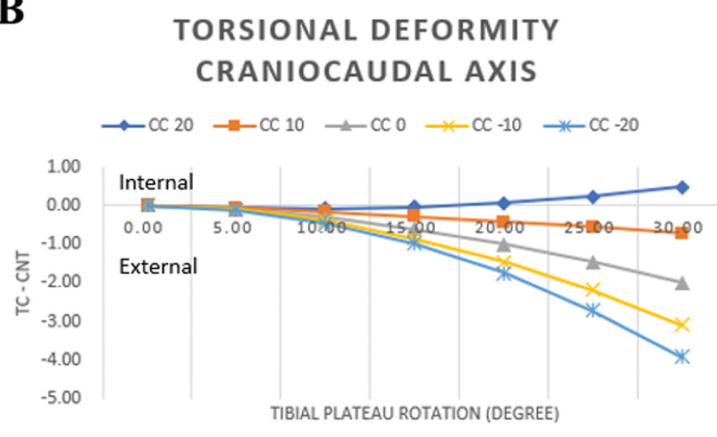

Fig 4. Torsional deformities in tibias that underwent osteotomies in the proximodistal (A) and craniocaudal directions (B). 


\section{Torsional deformity}

Internal rotation appeared in the tibias with osteotomies performed in the proximal-to-distal direction (PD, positive numbers; Fig 4). The tibias with osteotomies performed from the distal-to-proximal direction (PD, negative number) showed external rotation. The degree of rotational deformity increased with an increase in the magnitude of the osteotomy angle. Tibias with osteotomies performed in the cranial-to-caudal (CC, positive number) and caudal-to-cranial (CC, negative number) directions showed internal and external rotation tendencies, respectively, but they were within the normal range (2). The tibias with osteotomies performed in the proximalto-distal and distal-to-proximal directions showed the highest internal $\left(8.37^{\circ}\right)$ and external torsion $\left(-11.67^{\circ}\right)$, respectively. Torsional deformity of the tibia at $\mathrm{PD}=0 / \mathrm{CC}=0$ showed a minor change in the angle, which was in the range of $-1.99^{\circ}$ to $-0.10^{\circ}$.

\section{Discussion}

The present study provides evidence suggesting the tendency of occurrence of angular and torsional deformity with osteotomy along the proximodistal and craniocaudal directions in the CBLO. The maximum angular and torsional deformities were confirmed to be approximately $10^{\circ}$ in the craniocaudal and proximodistal directions, respectively. Therefore, our hypothesis that the deformity according to the magnitude of saw blade angulation will increase can be proved in this study.

Based on this study, it can consider correcting a preexisting angular or torsional deformity, but it should be avoided. Because of individual variations, it is difficult to apply the results of this study for correcting a surgery. In addition, the degree of deformity in each direction is different. For example, although the rotation angle was $30^{\circ}$ when tibial osteotomy was performed along the proximodistal direction, the mMPTA is in the normal range, but the variation in torsional deformity is extreme. This is similar to that in the case of tibia that underwent osteotomy in the craniocaudal direction. Therefore, there is a risk of developing another deformity while trying to correct one. Additionally, in a clinical situation, it is difficult to angulate in exactly one plane. If a surgeon performs an oblique plane osteotomy, it is difficult to predict the angular change.

Wheeler et al. reported a tendency of angular and torsional deformity with an increase in the angle of tibial plateau rotation with osteotomy along the proximodistal and craniocaudal directions in the TPLO. However, there were some cases in which the outcome did not correspond with the tendency of the angle size. In theory, for reduced osteotomies, the variation of angular or torsional deformity induced by tibial proximal segment rotation through an osteotomy angle along either the proximodistal or craniocaudal axes should be linearly related to both the degree of tibial proximal segment rotation and the angle of the osteotomy (21). The reasons for the deviations from the results of Wheeler et al. may be the differences between the surgical procedures, TPLO and CBLO. Both surgeries used a semicircular saw blade, but the saw positions were different. Therefore, the direction of deformity in each osteotomy is opposite to that of reported by Wheeler et al. Furthermore, Wheeler et al. performed manual tibial osteotomy and tibial proximal segment rotation, but in this study, it was performed more accurately using $3 \mathrm{D}$ computer software. Finally, this study could have yielded a more accurate result than did previous studies because unlike previous studies that used only one limb, this study used more limbs. These reasons may have contributed to the difference between the previous study and this study.

This study has several limitations, including the inheritable limitation of an in vitro study. 3D computer models of tibias were used in this study, and the actual characteristics of the patient's tibia, such as angular or torsional deformity, excessive TPA, and high or low insertion of tuberosity, may vary. The small sample size was another limitation of this study. A large case study may yield different results regarding both proximodistal and craniocaudal angulation. However, we were able to confirm a similar tendency in the four dogs; there may be some variation depending on the individual bone shape, but it is considered to show a similar tendency.

Lastly, we did not confirm the difference between the medial alignment reduction group and the complete reduction group, as in the case of a previous study (21). Reduction after leveling osteotomy may result in the alignment of the medial cortex rather than complete reduction. This is thought to be caused by the elimination of the gap to fit while applying the plate. In TPLO, angular and rotational deformities varied depending on the plateau rotation angle and reduction technique. The deformity was more severe in the alignment medial cortex group than in the complete reduction group. It was speculated that the tibial proximal segment was rotated while aligning the medial cortex, and consequently, the deformity is worsened. The CBLO is a radial osteotomy procedure like TPLO. Therefore, deformity will be more severe after performing reduction for aligning of the medial cortex. However, this limitation is beyond the scope of our study, and further studies are required to assess the effect of medial aligned cortex on the development of iatrogenic deformity during CBLO.

\section{Conclusion}

The results of this study revealed a tendency of occurrence of angular and torsional deformity with osteotomy along the proximodistal and craniocaudal directions in the CBLO. To the best of our knowledge, the present study makes a clinically significant contribution as the first report in veterinary medicine to confirm the deformities according to the magnitude of saw blade angulation. Further studies on the difference between the medial aligned cortex and complete reduction during plate application are needed.

\section{Acknowledgements}

This research was supported by University Innovation Support Project of Chungnam National University (2020-2021).

\section{References}

1. Apelt D, Kowaleski MP, Dyce J. Comparison of computed 
tomographic and standard radiographic determination of tibial torsion in the dog. Vet Surg 2005; 34: 457-462.

2. Aper R, Kowaleski MP, Apelt D, Tod Drost W, Dyce J. Computed tomographic determination of tibial torsion in the dog. Vet Radiol Ultrasound 2005; 46: 187-191.

3. Aragon CL, Budsberg SC. Applications of evidence-based medicine: cranial cruciate ligament injury repair in the dog. Vet Surg 2005; 34: 93-98.

4. Arnoczky S, Marshall J. The cruciate ligaments of the canine stifle: an anatomical and functional analysis. Am J Vet Res 1977; 38: 1807-1814.

5. Dismukes DI, Tomlinson JL, Fox DB, Cook JL, Song KJE. Radiographic measurement of the proximal and distal mechanical joint angles in the canine tibia. Vet Surg 2007; 36: 699704 .

6. Elkins A, Pechman R, Kearney M, Herron M. A retrospective study evaluating the degree of degenerative joint disease in the stifle joint of dogs following surgical repair of anterior cruciate ligament rupture. J Am Anim Hosp Assoc 1991; 27: 533-540.

7. Innes J, Bacon D, Lynch C, Pollard A. Long-term outcome of surgery for dogs with cranial cruciate ligament deficiency. Vet Rec 2000; 147: 325-328.

8. Jerram R, Walker A. Cranial cruciate ligament injury in the dog: pathophysiology, diagnosis and treatment. $\mathrm{N} \mathrm{Z} \mathrm{Vet} \mathrm{J}$ 2003; 51: 149-158.

9. Kim SE, Pozzi A, Kowaleski MP, Lewis DD. Tibial osteotomies for cranial cruciate ligament insufficiency in dogs. Vet Surg 2008; 37: 111-125.

10. Kishi EN, Hulse D. Owner evaluation of a cora-based leveling osteotomy for treatment of cranial cruciate ligament injury in dogs. Vet Surg 2016; 45: 507-514.

11. Korvick D, Johnson A, Schaeffer D. Surgeons' preferences in treating cranial cruciate ligament ruptures in dogs. J Am Vet
Med Assoc 1994; 205: 1318-1324.

12. Korvick D, Pijanowski G, Schaeffer D. Three-dimensional kinematics of the intact and cranial cruciate ligament-deficient stifle of dogs. J Biomech 1994; 27: 77-87.

13. Raske M, Hulse D, Beale B, Saunders WB, Kishi E, Kunze C. Stabilization of the CORA based leveling osteotomy for treatment of cranial cruciate ligament injury using a bone plate augmented with a headless compression screw. Vet Surg 2013; 42: 759-764.

14. Reif U, Hulse DA, Hauptman JG. Effect of tibial plateau leveling on stability of the canine cranial cruciate-deficient stifle joint: an in vitro study. Vet Surg 2002; 31: 147-154.

15. Slocum B, Devine T. Cranial tibial thrust: a primary force in the canine stifle. J Am Vet Med Assoc 1983; 183: 456-459.

16. Slocum B, Slocum TD. Tibial plateau leveling osteotomy for repair of cranial cruciate ligament rupture in the canine. Vet Clin North Am Small Anim Pract 1993; 23: 777-795.

17. Vasquez B, Hulse D, Beale B, Kerwin S, Andrews C, Saunders BW. Second-look arthroscopic findings after CORA-based leveling osteotomy. Vet Surg 2018; 47: 261-266.

18. Vasseur P. Clinical results following nonoperative management for rupture of the cranial cruciate ligament in dogs. Vet Surg 1984; 13: 243-246.

19. Vasseur P, Berry C. Progression of stifle osteoarthrosis following reconstruction of the cranial cruciate ligament in 21 dogs. $\mathrm{J}$ Am Anim Hosp Assoc 1992; 28: 129-136.

20. Warzee CC, Dejardin LM, Arnoczky SP, Perry RL. Effect of tibial plateau leveling on cranial and caudal tibial thrusts in canine cranial cruciate-deficient stifles: An in vitro experimental study. Vet Surg 2001; 30: 278-286.

21. Wheeler JL, Cross AR, Gingrich W. In vitro effects of osteotomy angle and osteotomy reduction on tibial angulation and rotation during the tibial plateau-leveling osteotomy procedure. Vet Surg 2003; 32: 371-377. 